\title{
Possibility of Splitting APS Dipoles to Make More ID Straights
}

\author{
formerly OAG-TN-2008-010 \\ Michael Borland-4/1/2008
}

\section{Accelerator Systems Division, Advanced Photon Source}

Recently we were asked by Ercan Alp if it would be possible to use three dipoles in an APS sector to make a second ID beamline possible, much as was done for the APSx3 design $[1,2]$. We've looked at this to see what might be possible without making too many changes to the ring. Ideally, we'd like to simply replace the one dipole with two shorter dipoles with an insertion device in the middle. Of course, we'd like to make the ID as long as possible, subject to limits on dipole strength, accelerator optics, and emittance.

We used elegant [3] to match an insertion device consisting of two sectors. The second (BM) dipole of the first sector was split at the $1 / 8^{\text {th }}$ point in terms of both angle and length. A zero length drift space was inserted that could then be stretched to create room for an ID. To simplify matters somewhat at this stage, we constrained the length of the two dipoles plus the drift to equal the original dipole length. I.e., we ignored for now the difference between arc and straight length. Our desire is to make the drift space as long as possible. If we limit the dipole field $B$ to $2 \mathrm{~T}$, then the total length of the dipoles must be greater than $L_{\text {min }}=H \theta / B$, where $H=23.35 \mathrm{Tm}$ is the rigidity and $\theta=\pi / 40-0.001$ is the total angle. $L_{\min }$ is therefore $0.91 \mathrm{~m}$, and hence the drift space can at most be $2.15 \mathrm{~m}$.

At least one, perhaps both, of the short dipoles will end up in a high-dispersion region, which will lead to emittance growth. Hence, we didn't want to drive the length too far and decided to limit it to $2 \mathrm{~m}$, approximately the same as in the APSx3 lattice. Hence, as in that lattice we should be able to accommodate a 1-m-long insertion device.

In addition to stretching the drift space, we allowed all twenty quadrupoles in the sectors to vary. Initial constraints included less than $20 \%$ deviation in lattice functions at the middle ID straight and less than $0.001 \%$ deviation in the final ID straight (to get a good match to the rest of the ring). We found the dispersion slope at the middle ID was quite hard to match, and made this a relatively loose constraint. We also had to increase the tolerance on changes in the horizontal beta function at the middle ID from 20 to $30 \%$. A constraint was also placed on the effective emittance to make it as small as possible.

Figure 1 shows the lattice functions for the two sectors. The magnet-to-magnet distance between the split dipoles is $1.97 \mathrm{~m}$, slightly short of the $2 \mathrm{~m}$ mark. The first dipole is $0.17 \mathrm{~m}$ long with a field of $1.35 \mathrm{~T}$, while the second dipole is $0.92 \mathrm{~m}$ long with a field of $1.73 \mathrm{~T}$.

The effective emittance assuming we formed a ring from such sectors is $4.95 \mathrm{~nm}$. Hence, if we add $N$ such modules to a ring with all other sectors being normal (3.13 nm effective emittance), the expected emittance is

$$
\frac{9.9 N+(40-2 N) 3.13}{40}
$$

. If we want to limit the emittance to $10 \%$ more than the nominal $3.13 \mathrm{~nm}$, we need $N<4$. If we limit it to under $4 \mathrm{~nm}$ (ESRF's emittance), then we have $N<10$.

Table 1 lists parameters of the new source point, assuming that we insert three such modules in the ring $(N=3)$ and that we have a $1.5 \%$ ratio of the raw emittances.

We attempted to improve the emittance and matching by allowing a gradient in the dipoles, thinking that perhaps it would provide additional optics flexibility. However, no improvement was obtained.

Several other issues remain with this scheme

1. The large dispersion slope at the downstream ID increases the effective emittance there to 3.7 $\mathrm{nm}$, even for a single insertion. 
Table 1: Parameters of new ID source point, assuming three such source points are inserted in the ring and $\epsilon_{y}=0.015 \epsilon_{x 0}$.

\begin{tabular}{|c|c|c|}
\hline Quantity & Value & Units \\
\hline$\beta_{x}$ & 1.64 & $\mathrm{~m}$ \\
\hline$\beta_{y}$ & 25.99 & $\mathrm{~m}$ \\
\hline$\alpha_{x}$ & 0.50 & \\
\hline$\alpha_{y}$ & 0.52 & \\
\hline$\eta_{x}$ & 0.049 & $\mathrm{~m}$ \\
\hline$\eta_{x}^{\prime}$ & -0.027 & \\
\hline$\sigma_{x}$ & 84.6 & $\mu \mathrm{m}$ \\
\hline$\sigma_{y}$ & 33.0 & $\mu \mathrm{m}$ \\
\hline$\sigma_{x}^{\prime}$ & 53.6 & $\mu \mathrm{rad}$ \\
\hline$\sigma_{y}^{\prime}$ & 1.4 & $\mu \mathrm{rad}$ \\
\hline
\end{tabular}

2. The vertical beta function at the new ID is very large, which will limit the ID gap. If we allow the same acceptance as for a \pm 2.5 -mm chamber in a regular straight section, then the chamber gap would have to be $\pm 5.6 \mathrm{~mm}$. This will limit the period and tuning range of the ID.

3. The large vertical beta function may present a beam instability issue with the new ID's transition pieces.

4. The radiation from the new ID will be accompanied by radiation from two strong dipoles. There is no possibility of introducing a Decker-type distortion [4] to soften this radiation, as there is no space. Hence, photon BPMs are not likely to work.

Issues 2 through 4 exist with the APSx3 lattice (see Figure 2 in [2]) as well. It is unclear if this scheme delivers enough to justify the emittance increase and other difficulties. It may depend on what photon energy the potential users need.

\section{References}

[1] M. Borland, "Possible Accelerator Upgrades," APS Upgrade Summary Workshop, August 10, 2006, http://www.aps.anl.gov/News/Conferences/2006/APS_Upgrade/SummaryWS.html

[2] V. Sajaev, M. Borland, and A. Xiao, "New Lattice Design for APS Storage Ring with Potential Tri-Fold Increase of the Number of Insertion Devices," PAC07, 1139-1141(2007); http://www.JACoW.org.

[3] M. Borland, "elegant: A Flexible SDDS-Compliant Code for Accelerator Simulation," Advanced Photon Source LS-287, September 2000.

[4] G. Decker and O. Singh, "Method for reducing x-ray background signals from insertion device x-ray beam position monitors," Phys. Rev. ST Accel. Beams 2, 112801 (1999). 


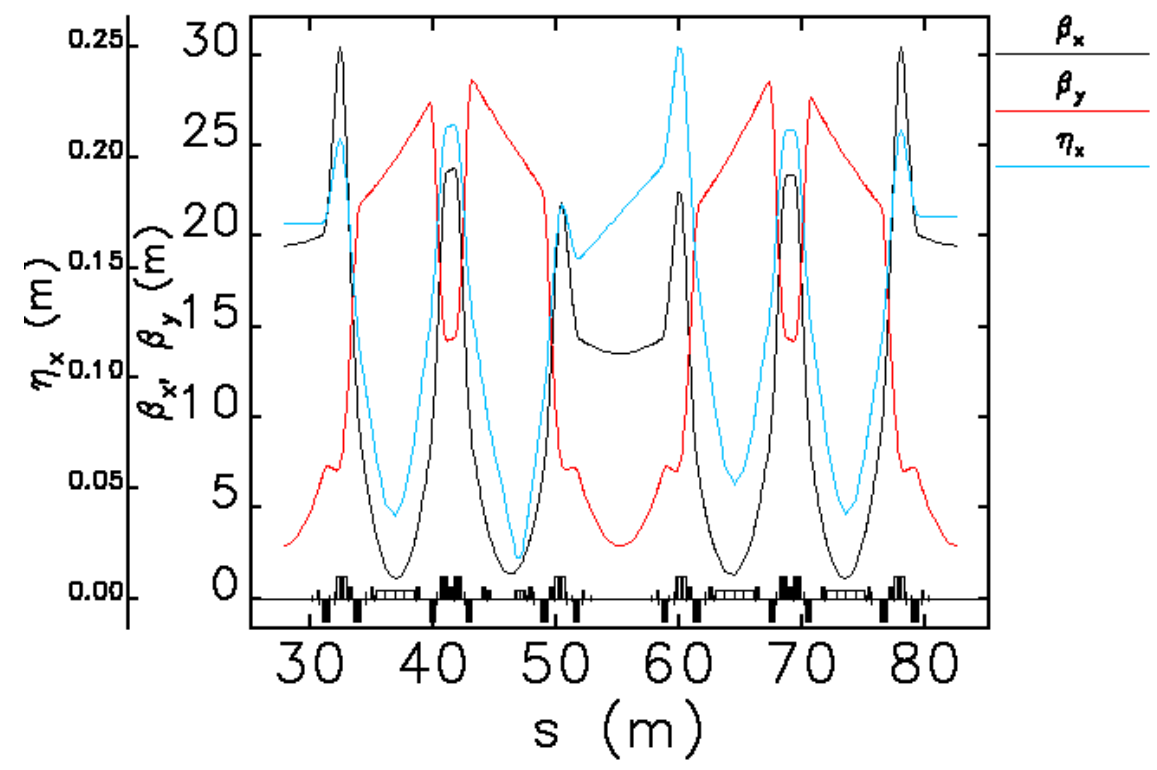

Figure 1: Lattice functions for the two sectors, including the sector (left half) with the split dipole. 